\title{
On the possible method of identification of two probably cognate Extensive Air Showers
}

\author{
Yu. Verbetsky ${ }^{a}$, M. Svanidze ${ }^{a}$, D. Beznosko ${ }^{b, *}$ and P. Homola ${ }^{c}$ \\ on behalf of the CREDO Collaboration \\ (a complete list of authors can be found at the end of the proceedings) \\ ${ }^{a}$ E. Andronikashvili Institute of Physics under Tbilisi State University, \\ 6 Tamarashvili st., Tbilisi, 0177 Georgia \\ ${ }^{b}$ Clayton State University, \\ 2000 Clayton State Blvd, Morrow, GA 30260, USA \\ ${ }^{c}$ Institute of Nuclear Physics Polish Academy of Sciences, \\ 152 Radzikowskiego st., Kraków, Poland \\ E-mail: yuri.verbetsky@iph.tsu.ge,manana.svanidze@tsu.ge, \\ dmitriybeznoskodclayton.edu, piotr.homola@ifj.edu.pl
}

\begin{abstract}
The persistent attempts are undertaken to show existence and investigate the special pairs of Extensive Air Showers (EAS) that can be suspected in common origin in the near space, i.e. to observe some consequence of existence of Cosmic Ray Ensemble (CRE). The remote cosmic ray stations observing EAS events are useful for this investigation. Such stations are operating within the GELATICA net (GEorgian Large-area Angle and TIme Coincidence Array) and are planed within the CREDO Collaboration (Cosmic Ray Extremely Distributed Observatory) as the CREDO-Maze project. The possible criteria are developed in the paper for detecting of two specific showers which ancestors have probable mutual proximity in their past.

Keywords: cosmic ray ensembles; large scale cosmic ray correlations; extensive air showers; pair of showers; relativistic invariant parameters; proximity definition.
\end{abstract}

37th International Cosmic Ray Conference (ICRC 2021)

July 12th - 23rd, 2021

Online - Berlin, Germany

"Speaker 


\section{Introduction}

Long-term researches of Primary Cosmic Radiation (PCR) properties have resulted in the need of investigation of pairs of remote Extensive Air Showers (EAS; or "showers") created by the PCR particles in the atmosphere and observed in widely spaced points. This new research guideline is connected with the obvious fact that some of these EAS pairs can be generated by cognate PCR particles created in a single process in the space. That is why the investigation of these related EAS couples generated by their PCR cognate ancestors can result in improvement of current notion of properties of the PCR (e.g. chemical composition), of properties of the near space medium (e.g. the medium density distribution) and possibly can support investigations of the Dark Matter properties [1,2]. Though only several shower-pair examples have been observed [3 - 8] so far. This poor data does not allow ascertainment of phenomenological properties of the flux of such pairs. All the more are inaccessible at present the investigations of the collective properties of their source - cognate PCR ancestor particles and their predecessors.

Whereas the poor present rate of observations of the (possibly) related EAS pairs is insufficient for research needs the big effective area of the remote Cosmic Ray (CR) stations for the observation of EAS events is desirable. Therefore the national networks of widely scattered small-scale CR stations, simple and inexpensive, are executing now the respective studies (e.g. ALTA, CZELTA, HiSPARC, LAAS, EEE, etc.).

The recently organized international association for the study of the problem, i.e. CosmicRay Extremely Distributed Observatory (CREDO [1,9]) includes, among others, some local CR networks with the CR stations possessing a capability to detect the arrival direction of the shower together with the shower's occurrence time. The CR stations with such capabilities are referred hereinafter as "EAS goniometers" or simply "goniometers". For instance the network GELATICA [10 - 15] in Georgia incorporates a set of goniometers located in the roof spaces of buildings of some universities in Tbilisi and Telavi. The system CREDO-Maze [1] with suitable properties is developing within the GREDO association.

At present it is necessary to design a selection method of the EAS pairs with showers generated by PCR particles, possibly spatially proximal in their past. Such EAS pairs can be preliminarily treated as the desired cognate EAS couples.

Hereinafter the special relativistic invariant kinematic quantities are designed for this purpose. All available data from two remote goniometers estimating motion characteristics of two EAS observed are measured in common inertial reference frame and used completely for this purpose. These special quantities can be used to establish the spatial proximity of the ancestor particles of both showers in their past. The dimensionless verifying parameters are proposed for extraction of the desired historically proximal EAS couples from the sample of the observed random EAS pairs.

\section{Problem statement and main definitions}

Let us accept for the required measure of the possible historical proximity of two showers the minimal distance between the two moving ancestor CR particles, which must be interpreted with consideration of estimation error. Let us denote by $\mathbf{r}_{01}, \mathbf{r}_{02}$ the radius-vectors of the observed remote shower cores and by $\mathbf{M}_{1}, \mathbf{M}_{2}$ the respective covariance matrices. Let it $t_{01}^{\prime}, t_{02}^{\prime}$ and 
$\sigma_{\mathrm{t} 1}{ }^{2}, \sigma_{\mathrm{t} 2}{ }^{2}$ be the times of the cores observations and the dispersions of their estimations. Finally let us denote by $\mathbf{n}_{1}, \mathbf{n}_{2}$ the unit dimensionless vectors describing the showers' moving directions, with $\mathbf{D}_{1}, \mathbf{D}_{2}$ being the covariance matrices of their estimations. This is a complete set of values directly available and measured by a pair of goniometers. But if the coordinates of the shower cores $\mathbf{r}_{01}, \mathbf{r}_{02}$ are not available for simple goniometers, the proper coordinates of the CR stations themselves can be used instead, if the distance between the installations is sufficiently big. The theoretical estimation of average spread of the possible shower cores around the goniometer's center can be used in this case as estimation of the $\mathbf{r}_{01}, \mathbf{r}_{02}$ coordinates' uncertainty.

For handy determination of the required measure of occured possible proximity of two showers under consideration let us associate every EAS with a moving point coinciding with some accuracy with the center of the shower's core and its acestor particle. It can be accepted that this representing point is moving rectilinearly with the light speed $c$ in the near space, as the influence of existing external fields is negligible for particles of very high energy. So its travel is described approximately by an equation of uniform motion with the $c$ velocity.

Let us accept the average time $t_{c}^{\prime}=\left(t_{02}^{\prime}+t_{01}^{\prime}\right) / 2$ of two measured times of showers' observation for the system time origin. So the time of any event is defined now as $t=t^{\prime}-t_{\mathrm{c}}^{\prime}$ and both showers' observation times are $t_{1}=t_{01}^{\prime}{ }-t_{\mathrm{c}}^{\prime}=-\delta t / 2, t_{2}=t_{02}^{\prime} t^{\prime}{ }_{\mathrm{c}}=\delta t / 2$. Obviously the time difference $\delta t$ is of any sign. The showers observation times' absolute values $\left|t_{1}\right|,\left|t_{2}\right|$ are equal, and the moments themselves are opposite in sign. So the earliest observation occurs at negative system time.

\section{Kinematics of two showers' ancestors motion}

Surely the variable vector connecting two moving representing points $\mathbf{r}_{1}(t), \mathbf{r}_{2}(t)$ at the moment $t$ depends linearly on this running time:

$$
\Delta(t)=\mathbf{r}_{2}(t)-\mathbf{r}_{1}(t)=[\delta \mathbf{r}-<\mathbf{n}>(c \cdot \delta t)]+\delta \mathbf{n} \cdot c t
$$

Here $\delta \mathbf{r}=\left(\mathbf{r}_{02}-\mathbf{r}_{01}\right)$ is the vector connecting both points of the shower cores' observation, while $\delta \mathbf{n}=\left(\mathbf{n}_{2}-\mathbf{n}_{1}\right)$ is the difference vector of the showers' velocity unit vectors. The respective average vector is denoted by $\left\langle\mathbf{n}>=\left(\mathbf{n}_{2}+\mathbf{n}_{1}\right) / 2\right.$. The last two vectors are mutually orthogonal $(<\mathbf{n}>\mathrm{T} \cdot \delta \mathbf{n})=0$. The dispersions and covariance matrixes of specified secondary values and vectors can be immediately calculated as the initial values concerning remote showers are statistically independent: $\sigma_{\delta \mathrm{t}}{ }^{2}=\sigma_{\mathrm{t} 1}{ }^{2}+\sigma_{\mathrm{t} 2}{ }^{2}$ for the observation times' difference; $\mathbf{M}=\mathbf{M}_{1}+\mathbf{M}_{2}$ for the shower cores' observation coordinates difference; $\mathbf{D}=\mathbf{D}_{1}+\mathbf{D}_{2}$ for the difference vector of the showers' velocity unit vectors and $\left(\mathbf{D}_{1}+\mathbf{D}_{2}\right) / 2^{2}=\mathbf{D} / 4$ for the respective average vector.

The smallest length of the difference vector (1) during the whole period of the showers' representing points' motion will be assumed as the source for design of the desired measure of the showers' historical proximity.

For this propose let us use the relativistic invariant quantity, namely the squared interval between the moving representing points of both showers. The spatial vector $\Delta(t)$ connecting the two moving points (1) at the same time is known. As the time difference between these two relativistic events is zeroth-order by definition in the reference frame used, the squared interval $s^{2}(t)=-(c \cdot 0)^{2}+\boldsymbol{\Delta}(t)^{\mathrm{T}} \cdot \boldsymbol{\Delta}(t)$ is spacelike one. [For our purposes, the form $(-1,+1,+1,+1)$ of Minkowski's metric tensor is convenient.] 
This squared interval has the smallest value at the moment

$$
\tau=-(1 / c) \cdot\left(\delta \mathbf{r}^{\mathrm{T}} \cdot \delta \mathbf{n}\right) /\left(\delta \mathbf{n}^{\mathrm{T}} \cdot \delta \mathbf{n}\right) .
$$

If the moment $\tau$ is negative, the representing points have been in the nearest position in the past of the two-shower reference frame. If $\tau>0$ the representing points seem approaching each other in future, although they are absorbed in the underlying ground.

The variable connecting vector (1) at the moment $\tau$ of the points' closest approach (2) obtains the value: $\quad * \Delta=\boldsymbol{\Delta}(\tau)=\delta \mathbf{r}-<\mathbf{n}>(c \cdot \delta t)-\delta \mathbf{n} \cdot\left(\delta \mathbf{r}^{\mathrm{T}} \cdot \delta \mathbf{n}\right) /\left(\delta \mathbf{n}^{\mathrm{T}} \cdot \delta \mathbf{n}\right)$

In the figure 1 the special case of simultaneous motion of two representing points along two straight lines is shown, i.e. the closest approach of the points accomplished before the earliest observation of one of the showers.

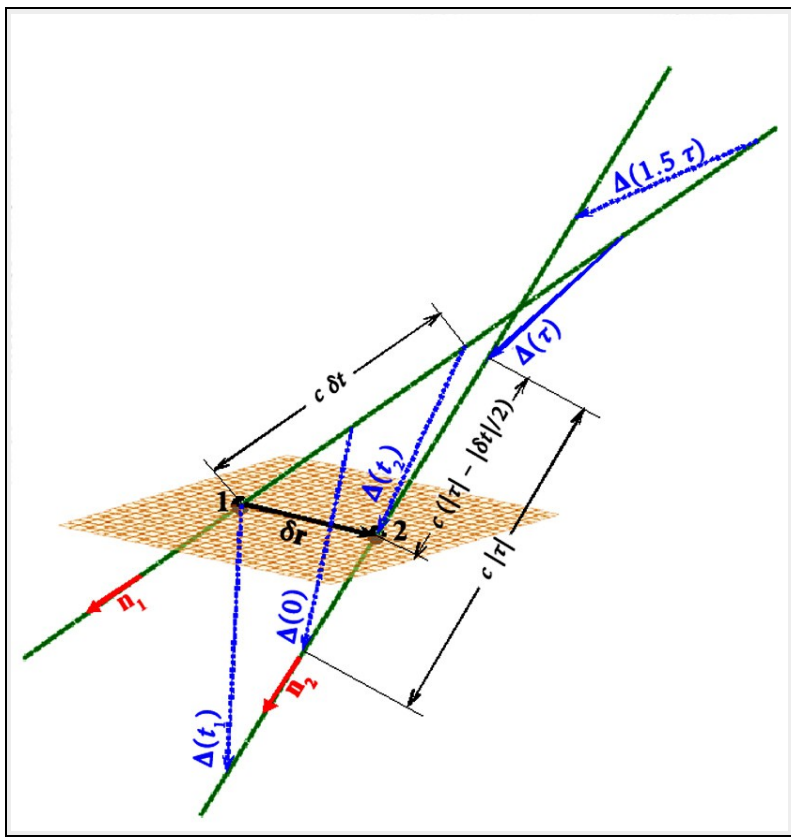

Figure 1 The scheme of motion of two representing points.

The trajectories of the representing points are shown in green.

Symbols $\mathbf{1}$ and $\mathbf{2}$ indicate observation points of the shower cores. The motion directing unit vectors of both representing points are shown in red.

The vectors connecting the representing points at the specified moments are shown in blue. Some lengths of the characteristic segments of trajectories are specified too.

Consequently the respective relativistic invariant squared interval $s^{2}(t)$ obtains the minimal value ${ }^{*} s^{2}=s^{2}(\tau)=\boldsymbol{\Delta}(\tau)^{\mathrm{T} \cdot \boldsymbol{\Delta}(\tau)}$ at the moment $t=\tau$. As this value is independent of the choice of the inertial reference frame so the dispersion $\sigma_{\mathrm{s} 2}^{2}$ of its estimate can be determined in the same reference frame. This quantity describes the estimation precision of nonvarying value ${ }^{*}{ }^{2}$, so it is invariant too. It can be calculated explicitly and the respective rather cumbersome formula is:

$$
\sigma_{\mathrm{s} 2}^{2}=4\left({ }^{*} \boldsymbol{\Delta}^{\mathrm{T}} \cdot<\mathbf{n}>\right)^{2} \cdot\left(c^{2} \cdot \sigma^{2} \delta \mathrm{t}\right)+{ }^{*} \boldsymbol{\Delta}^{\mathrm{T}} \cdot\left[4\left(\boldsymbol{\Pi}^{\mathrm{T}} \cdot \mathbf{M} \cdot \boldsymbol{\Pi}+\mathbf{G}^{\mathrm{T}} \cdot \mathbf{D} \cdot \mathbf{G}\right)+(c \cdot \delta t)^{2} \cdot \mathbf{D}\right] \cdot * \boldsymbol{\Delta}
$$

Here the auxiliary matrixes are used:

$$
\begin{aligned}
& \boldsymbol{\Pi} \sim\left[\Pi^{\mu}{ }_{v}\right]=\left[\delta^{\mu}{ }_{v}\right]-\left[\delta n^{\mu} \delta n_{v}\right] /\left(\delta n^{\alpha} \delta n_{\alpha}\right) ; \mu, v, \alpha, \ldots=x, y, z \\
& \mathbf{G} \sim\left[G^{\mu}{ }_{v}\right]=\left\{\left[\delta n^{\mu} \delta n_{v}\right] /\left(\delta n^{\alpha} \delta n_{\alpha}\right)+\left[\delta^{\mu}{ }_{v}-2\left[\delta n^{\mu} \delta n_{v}\right] /\left(\delta n^{\alpha} \delta n_{\alpha}\right)\right]\left(\delta r^{\beta} \delta n_{\beta}\right) /\left(\delta n^{\alpha} \delta n_{\alpha}\right)\right\}
\end{aligned}
$$

(the usual rule of summation over repeated index is implied)

We shall use the value of Lorentz-invariant space-like interval

$$
\Delta .=\left(* \Delta^{\mathrm{T}} * \Delta\right)^{1 / 2}
$$

between the representing points at the moment of their closest approach $\tau$ as a measure of the historical proximity of the showers of the given pair. The dispersion of this quantity is equal to $\sigma_{\Delta}^{2}=\sigma_{\mathrm{s} 2}^{2} /(2 \Delta .)^{2}$. 


\section{Definition of two verifying parameters and of a criterion of the two showers' historical proximity}

If the ancestors of a pair of showers are suspected in common origin, they must possess the following properties:

- Both representing points have approached with each other before the observation of the earliest shower by one of the goniometers, i.e. $\tau<\min \left(t_{1}, t_{2}\right)=-|\delta t / 2|<0$.

- The estimation of minimal value $\Delta$. of the interval between the representing points of both ancestors of the observed showers has nonsignificant difference with anticipated zero distance.

All possible time intervals connected with the system of two showers' under investigation we shall compare with the light propagation time between the observation points of the shower cores, i.e. $|\delta \mathbf{r}| / c$. The difference between the observation time of the earliest of the shower cores and the moment of the ancestors' closest approach can be described by the dimensionless ratio:

$$
\left(\tau-\min \left(t_{1}, t_{2}\right)\right) /(\mid \delta \mathbf{r} / / c)=c \cdot(\tau+|\delta t / 2|) /|\delta \mathbf{r}|
$$

Since this is a ratio of two time periods measured in the same reference frame, both periods are changed by the same gamma-factor under any Lorentz transformation and the ratio behaves as the dimensionless relativistic invariant.

The last ratio can attain large numerical values of either sign. This property makes difficulties in pictorial view of all possible time sequencing. As a usual logarithm scale is unsuitable for negative values, the well-known function of inverse hyperbolic sine can be used instead. This function behaves asymptotically as the logarithmic one but preserves the argument sign and is nearly linear in the null argument vicinity. That is why it is convenient to define the time Sequencing dimensionless parameter $S$ for two showers under consideration by the expression:

$$
S=\operatorname{arsinh}(c \cdot(\tau+|\delta t / 2|) /|\delta \mathbf{r}|) ;-\infty<S<\infty
$$

Any pair of showers whose ancestors approached in the past is characterized by a negative value of the verifying parameter $S$. The positive $S$ value means that the showers' ancestors approach to each other after observation of the earlier of two showers, but in fact it appears that they get absorbed in the ground under the goniometers.

In the case of generation of two ancestor particles of the showers under consideration in a single center, the value of $\Delta$. turns out to be a real estimate of the value of the former spatial proximity of these particles. The statistical significance of the deviation of this value from the assumed zero distance can be described by the ratio $\Delta_{\mathbf{v}} / \sigma_{\Delta}$. The smaller is this value, the more reliable is the statement about the close proximity of two ancestor particles that took place in their distant past. With respect to the two showers observed, this closeness took place in their historical past. This non-negative ratio can, however, attain large values. Therefore, it is convenient to determine the parameter $P$ of the historical Proximity of two observed showers by the dimensionless value:

$$
P=-\ln \left(\Delta_{\mathbf{*}} / \sigma_{\Delta} \cdot k\right) ; \quad-\infty<P<\infty
$$

The optional factor $k$ adjusts the strictness of the historical proximity definition. The possible increase of the factor $k$ enlarges the number of "possibly historically proximal showers" in the sample of shower pairs under investigation, but the pairs with more and more doubtful proximity occur "proximal" meanwhile. It is just the reason why the choice of optional coefficient $k$ is the matter of compromise and consequently the matter of agreement. 
Since the historical proximity parameter $P$ is a function of two Lorentz-invariants it is relativistic invariant quantity itself.

Any historically proximal couple (take into account the optional choice of the factor $k$ ) of showers has positive verifying parameter $P$. The larger is the estimated value of $P$ the more reliable is the statement of anticipated historical proximity of both showers.

Two verifying dimensionless parameters $(4,5)$ define all possible cases of relationship between the ancestors of showers in the pair. The whole $(P, S)$ plane can be divided into three distinctive zones as is shown in the figure 2.

Figure 2. The plane of verifying invariant parameters for the pair of extensive air showers

- Zone 1 consists of pairs of showers with representing points which can approach one another in the future, but have to be absorbed in the ground: $S>0$;

- Zone 2 consists of pairs of showers with representing points which have been close in their past, but the estimation precision prevents the decision of their historical proximity: $S<0, P<0$;

- Zone 3 consists of pairs of showers with representing points approaching closely in their past and propagating from a single point

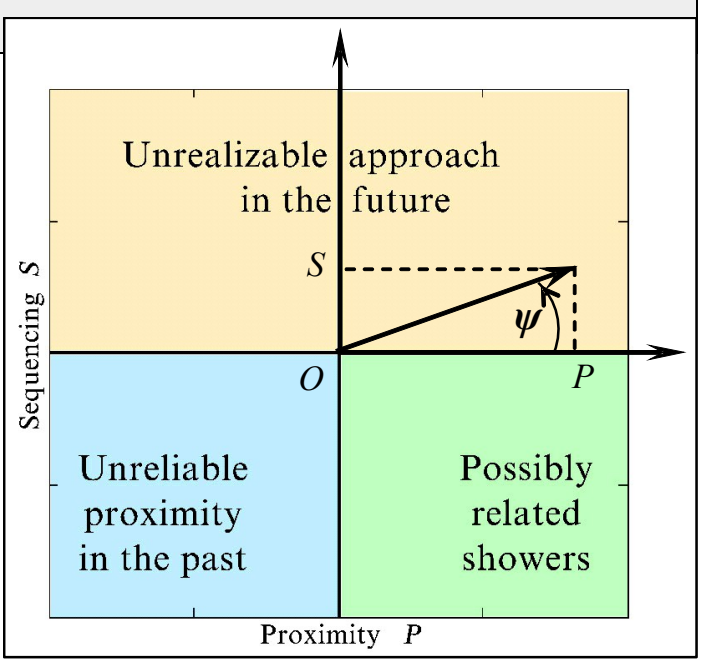
within the estimation precision under preferred strictness factor $k$ value: $S<0, P>0$.

Any point in the $(P, S)$ plane defines the angle $\psi$ (figure 2) between $O P$ axis and the direction from the origin $O$ to the specified $(P, S)$ point. This angle marks out belonging of the specified point to some of the three zones. Consequently we can define combined verifying criterion as

$$
K=(2 / \pi) \cdot \arctan (S / P) ; \quad 0<K<4
$$

The value of criterion $K$ specifies belonging of the given $(P, S)$ point to:

Zone 1: $0<K<2$ for unrealizable approach in the future;

Zone 2: $2<K<3$ for unreliable proximity in the past;

Zone 3: $3<K<4$ for possibly historically related showers.

The illustrative integer bounds of these three $K$-zones delimit the quadrants of the $(P, S)$ parameters' plane (figure 2). The combined criterion $K$ is a relativistic invariant too. It allows a plain ascertainment of acceptability of the assumption that the ancestors of the pair of showers in question have been spatially proximal in their past within the selected relative accuracy, i.e. the obseved showers themselves are historically proximal.

\section{Conclusion}

The suggested pair of Lorentz-invariant $(P, S)$ parameters $(4,5)$ can be useful for the classification of the pairs of showers observed in the remote points for evidence of the spatial proximity of their ancestors in their history and of the possibility of their common origin. The 
combined (6) criterion $K$ is useful for the preliminary selection of the suitable historically proximal couple from the initial sample of the remote EAS pairs: once the shower couple with the $K$ value within the $3<K<4$ range is found out it must be thoroughly investigated. Meanwhile the optional factor $k$ in the historical Proximity $P$ definition (4) adjusts the strictness of the required couples' selection.

The subsequent study of the selected couple's properties can examine the obtainable kinematic parameters, that is the time (2) of the closest approach moment $\tau$ of the showers' ancestors and the value (3) of the space-like interval $\Delta$. between them at the $\tau$ moment. They are the available estimators (alongside with their dispersions) for investigation of the possible physical connection between the showers' ancestors of the pair. The historical proximity of the showers of the couple is a necessary consequence of the real uniqueness of their ancestors' origin; but the historical proximity of the showers of the pair established by the use of $(P, S)$ and $K$ parameters is not itself the sufficient cause for conclusion of common origin of their ancestors.

\section{References}

[1] Homola, P., et al.. "Cosmic-ray extremely distributed observatory". Symmetry, (2020), 12(11), pp.1835-1894. https://www.mdpi.com/2073-8994/12/11/1835 Cosmic-Ray Extremely Distributed Observatory (CREDO): http://credo.science/

[2] Warsaw Workshop on Non-Standard Dark Matter (2016): http://indico.fuw.edu.pl/conferenceDisplay.py?confId=45

[3] Ochi N., Iyono A., Kimura H. et al. "Search for large-scale coincidences in network observation of cosmic ray air showers". J. Phys G: Nuclear and Particle Physics (2003) 29, №6, pp.1169-1180.

[4] Atsushi Iyono, Hiroki Matsumoto, Kazuhide Okei et al:

"Parallel and Simultaneous EAS events due to Gerasimova-Zatsepin effects observed by LAAS experiments"; proceedings of the $31^{\text {st }}$ ICRC (2009), Łódź; http://icrc2009.uni.lodz.pl/proc/pdf/icrc0941.pdf

[5] Karel Smolek, Filip Blaschke, Jakub Čermak et al: "ALTA/CZELTA - a sparse very large air shower array: overview of the experiment and first results"; proceedings of the $31^{\text {st }}$ ICRC (2009), Łódź; http://icrc2009.uni.10dz.pl/proc/pdf/icrc1300.pdf

[6] F. Blaschke, J. Čermák, J. Hubík et al: "CZELTA: An overview of the CZECH large-area time coincidence array"; Astrophys. Space Sci. Trans. (2011), 7, pp.69-73; www.astrophys-space-sci-trans.net/7/69/2011.

[7] Yu. Verbetsky, M. Svanidze, A. Iashvili, E. Tskhadadze and D. Kokorashvili: "First results on the spatiotemporal correlations of the remote Extensive Air Shower pairs". $23^{\text {rd }}$ ECRS (and 32 $2^{\text {nd }}$ RCRC) Moscow, J. Phys.: Conf. Ser. 409 (2013) 012085; http://iopscience.iop.org/article/10.1088/17426596/409/1/012085/meta;jsessionid=89322C00C25D03FA0B0D63C56F0B2333. C5.iops cience.cld.iop.org

[8] M. Abbrescia, L. Baldini, R. Baldini Ferroli et al.: "Search for long distance correlations between extensive air showers detected by the EEE network", Eur. Phys. J. Plus (2018) 133 p.34

[9] Piotr Homola for the CREDO Collaboration, "Invitation to the Comic Ray Extremely Distributed Observatory" (ID: \#1448, published in this conference)

[10] GELATICA network: http://www.gelatica.tsu.ge/ 
[11] Yu Verbetsky, M Svanidze, A Iashvili, E Tskhadadze and D Kokorashvili.

"First results on the spatiotemporal correlations of the remote Extensive Air Shower pairs".

23rd ECRS (and 32nd RCRC) Moscow (2012) J. Phys.: Conf. Ser. 409 (2013) 012085

http://iopscience.iop.org/article/10.1088/17426596/409/1/012085/meta; jsessionid=89322C00C25D03FA0B0D63C56F0B2333.C5.iops cience.cld.iop.org

[12] Yuri Verbetsky, Manana Svanidze, Abesalom Iashvili, Levan Kakabadze: “Extensive Air Showers' Arrival Direction Distribution by TBS Array”, International Journal of High Energy Physics.(2014) 1, №4, pp.49-54, http://www.sciencepublishinggroup.com/journal/paperinfo? journalid=124\&doi=10.11648/j.ijhep.20140104.11

[13] Manana Svanidze, Yuri Verbetsky, Ia Iashvili, et al: “Angular distribution of extensive air showers by TEL array under GELATICA experiment”; GESJ: Physics (2016) №1(15) pp.54-62; http://gesj.internet-academy.org.ge/download.php?id=2740.pdf

[14] Yu. G. Verbetsky, M. S. Svanidze, A. Iashvili, I. Iashvili, L. Kakabadze: "Extensive air showers' arrival direction distribution by the TSU array under GELATICA experiment"; GESJ: Physics (2018) №2(20) pp.43-55;

http://gesj.internet-academy.org.ge/download.php?id=3175.pdf

[15] Yuri Verbetsky, Manana Svanidze, Abesalom Iashvili, Ia Iashvili, Levan Kakabadze, Nino Jonjoladze: "Investigation of the arrivals' directions differences for consecutive Extensive Air Showers using the data taken by TEL goniometer under GELATICA network", GESJ: Physics (2019) №2(22) pp.27-33;

http://gesj.internet-academy.org.ge/download.php?id=3274.pdf 


\section{Full Authors List: CREDO Collaboration}

Oleksandr Sushchov $^{1}$, Piotr Homola ${ }^{1}$, David E Alvarez Castillo ${ }^{1,2}$, Dmitriy Beznosko ${ }^{3}$, Nikolai Budnev ${ }^{4}$, Dariusz Góra $^{1}$, Alok C Gupta ${ }^{5}$, Bohdan Hnatyk ${ }^{6}$, Marcin Kasztelan ${ }^{7}$, Peter Kovacs ${ }^{8}$, Bartosz Łozowski ${ }^{9}$, Mikhail V Medvedev ${ }^{10,11}$, Justyna Miszczyk ${ }^{1}$, Alona Mozgova ${ }^{6}$, Vahab Nazari ${ }^{2,1}$, Michal Niedzwiecki ${ }^{12}$, Maciej Pawlik ${ }^{13,14}$, Matías Rosas ${ }^{15}$, Krzysztof Rzecki ${ }^{14}$, Katarzyna Smelcerz ${ }^{12}$, Karel Smolek ${ }^{16}$,

Jaroslaw Stasielak $^{1}$, Slawomir Stuglik ${ }^{1}$, Manana Svanidze ${ }^{17}$, Arman Tursunov ${ }^{18}$, Yuri Verbetsky ${ }^{17}$, Tadeusz Wibig ${ }^{19}$, Jilberto Zamora-Saa ${ }^{20}$.

${ }^{1}$ Institute of Nuclear Physics Polish Academy of Sciences, Radzikowskiego 152, 31-342 Kraków, Poland.

${ }^{2}$ Joint Institute for Nuclear Research, Dubna, 141980 Russia.

${ }^{3}$ Clayton State University, Morrow, Georgia, USA.

${ }^{4}$ Irkutsk State University, Russia.

${ }^{5}$ Aryabhatta Research Institue of Observational Sciences (ARIES), Manora Peak, Nainital 263001, India.

${ }^{6}$ Astronomical Observatory of Taras Shevchenko National University of Kyiv, 04053 Kyiv, Ukraine.

${ }^{7}$ National Centre for Nuclear Research, Andrzeja Soltana 7, 05-400 Otwock-Swierk, Poland.

${ }^{8}$ Institute for Particle and Nuclear Physics, Wigner Research Centre for Physics, 1121 Budapest, Konkoly-Thege Miklós út 29-33, Hungary.

${ }^{9}$ Faculty of Natural Sciences, University of Silesia in Katowice, Bankowa 9, 40-007 Katowice, Poland.

${ }^{10}$ Department of Physics and Astronomy, University of Kansas, Lawrence, KS 66045, USA.

${ }^{11}$ Laboratory for Nuclear Science, Massachusetts Institute of Technology, Cambridge, MA 02139, USA.

${ }^{12}$ Department of Computer Science, Faculty of Computer Science and Telecommunications, Cracow University of Technology,Warszawska, 24, 31-155 Kraków, Poland.

${ }^{13}$ ACC Cyfronet AGH-UST, 30-950 Kraków, Poland.

${ }^{14}$ AGH University of Science and Technology, Mickiewicz Ave., 30-059 Kraków, Poland.

${ }^{15}$ Liceo 6 Francisco Bauzá, Montevideo, Uruguay.

${ }^{16}$ Institute of Experimental and Applied Physics, Czech Technical University in Prague.

${ }^{17} \mathrm{E}$. Andronikashvili Institute of Physics under Tbilisi State University, Georgia.

${ }^{18}$ Research Centre for Theoretical Physics and Astrophysics, Institute of Physics, Silesian University in Opava, Bezrucovo nám. 13, CZ-74601 Opava, Czech Republic.

${ }^{19}$ University of Łódz, Faculty of Physics and Applied Informatics, 90-236 Łódz, Pomorska 149/153, Poland.

${ }^{20}$ Universidad Andres Bello, Departamento de Ciencias Fisicas, Facultad de Ciencias Exactas,

Avenida Republica 498, Santiago, Chile. 